\title{
Triple-Polarized Multi-User Mimo-Idma System under Correlated Fading Channel
}

\author{
B. Partibane ${ }^{1}$, V. Nagarajan ${ }^{2}$ \\ ${ }^{1}$ Department of ECE, SSN College of Engineering, Chennai, India \\ ${ }^{2}$ Department of ECE, Adhiparasakthi Engineering College, Melmaruvathur, India \\ Email: partibaneb@ssn.edu.in
}

Received 31 March 2016; accepted 7 April 2016; published 16 June 2016

Copyright (C) 2016 by authors and Scientific Research Publishing Inc.

This work is licensed under the Creative Commons Attribution International License (CC BY).

http://creativecommons.org/licenses/by/4.0/

c) (i) Open Access

\section{Abstract}

A multiple-input multiple-output interleave division multiple access (MIMO-IDMA) system with Triple Polarized Division Multiplexing (TPDM) is presented in this paper. The present methodology replaces three independent linearly polarized antennas with a single triple polarized antenna at both the transmitter and receiver. The users in the communication link are accommodated and separated using a user-specific interleaver combined with low rate spreading sequence. To eliminate the effects of multi-stream interference (MSI), minimum mean square error (MMSE) algorithm based on successive interference cancellation (SIC) Multi-user detection (MUD) technique is employed at the receiver. Furthermore, log-maximum a posteriori probability (MAPP) decoding algorithm is implemented at the mobile stations (MSs) to overcome the effects of multi-user interference (MUI) effects. The paper also evaluates the effects of coded MIMO-IDMA in the downlink communication by adopting the Stanford University Interim (SUI) and Long-term Evolution (LTE) channel model specifications. In comparison with the traditional uncoded system, the present solution considering turbo coded triple-polarized MIMO-IDMA system with iterative decoding algorithm provides better bit error rate (BER) with reduced signal to noise ratio (SNR). The simulation results also show that though the SNR requirement is higher for the proposed technique compared to the conventional uni-polarized antenna based MIMO-IDMA system, it gives the advantages of achieving higher data rate with reduced cost and space requirements in the context of a downlink (DL).

\section{Keywords}

Interleave Division Multiple Access (IDMA), Multiple Input Multiple Output (MIMO), Multi-User Interference (MUI), Stanford University Interim (SUI) Channel Model, Long Term Evolution (LTE) Channel Model 


\section{Introduction}

The triple-polarized multiple-input multiple-output (MIMO) with interleave division multiple access (IDMA) techniques has evolved as the next generation wireless system as it supports higher data rate with reduced inter symbol interference (ISI) and MAI. In a conventional IDMA system, linearly polarized antennas are exploited to establish the communication link. Further, it has also been demonstrated that the capacity of the channel can be improved by increasing the number of antennas used in the communication system. This increases the diversity gain of the antenna. Further to minimize the coupling effects between the antennas, the radiating elements are spaced at least half a wavelength at the subscriber station and ten times the wavelength in case of the base stations [1]. This assures maximum capacity and reliable benefits achievable in a MIMO-IDMA wireless system. This poses stringent specifications on the infrastructure resulting in increased cost. Therefore this paper aims to overcome this limitation through the usage of triple-polarized antennas for MIMO-IDMA system.

The utility of circularly polarized (CP) antenna arrays for MIMO is demonstrated in [2]. The CP antennas in the single input single output (SISO) system presented in [2] performed better than the single linearly polarized antenna in terms of spectral efficiency in multipath fading environments. The IDMA system proposed in [3] exploited the use of user specific interleavers combined with low rate channel coding for user separation. This is in contrast to the code division multiple access (CDMA) scheme proposed in [4] where the users are separated using user specific spreading sequences. Though CDMA and IDMA systems have the potential of delivering higher diversity gain and mitigating the inter-cell interference, IDMA has specific advantages over CDMA. This includes the usage of low complex multiuser detectors than required for CDMA systems [5]-[12]. Further studies in [4] [5] [13]-[18] have proven that IDMA systems can outperform CDMA when iterative (turbo) receivers are used. Therefore recent developments have shown that IDMAs can be easily integrated into the MIMO systems [15]. The interleaver for use in IDMA systems is designed and discussed in [16] and [17].

The performance of the MIMO system in SM or TD relies on the channel characteristics which are more closely related to the antenna parameters such as the antenna height, spacing and scattering effects [9]. Since the multiplexing gain or diversity gains are the functions of the number of antennas used in the system, more antennas are highly preferred. However increasing the number of antennas poses challenge in terms of space requirements since at least ten times the wavelength is the minimum required spacing between the antenna elements in case of a base station scenario. Also, the number of antennas is restricted to 4 and lesser due to size and energy considerations [19].

The importance of the IDMA system and its benefits in a MIMO system are described in [3]-[7]. A single antenna based IDMA system employing BPSK-modulation and the corresponding channel estimation is studied in [18]. Further an extended IDMA system called multi-layer IDMA is proposed in [19]. The integration of orthogonal frequency-division multiplexing (OFDM) with IDMA and its benefits are discussed in [19]-[21].

In the aforementioned literature works, most of the research adopted a dual-polarised antenna system in the context of a single user communication system to achieve SM and TD. The impact of the number of users on the performance of such systems remains unexplored since the increase in the number of users introduces additional design challenges such as the MUI [22]-[25]. Multi-user detection (MUD) techniques are employed at the BS and MS to overcome the effect of MUI. But the limiting factor is the complexity involved in the detection scheme [6]. Therefore to overcome this limitation, transmitter side pre-processing technique to be called as transmitter pre-processing (TP) or multi-user TP (MUTP) is employed in DL communications. Hence, the TP along with the receiver side post processing enables to overcome MUI and inter-antenna interference (IAI) in a DL-MIMO system [6] [22]-[24]. The performance of this pre-processing technique has been investigated in time division duplex (TDD) system [6].

In a multi-user system, high performance gain can be realized using iterative turbo style interaction between the detector and channel decoder [26] [27]. In [3] [5] and [7], the iterative IDMA receiver designs have resulted in minimum mean square error and soft rake equalizers. On the other hand, these designs are limited at the receiver to a single antenna system or to the BPSK modulation scheme or to the perfect channel state information (CSI). The analysis on the effect of coupling in a dual polarized MIMO channel is discussed in [28]-[31]. The channel imperfections such as the delay spread, ricean $\mathrm{K}$ factor and cross polarization discrimination (XPD) also affects the channel capacity which is proven through outdoor measurements as demonstrated in [25]. The dualpolarized MIMO system exploiting space time block is studied in [26] and the test bed mimicking urban radio communication is demonstrated in [28]. Furthermore the receiver cooperation in a Gaussian MIMO channel [29], 
the channel state information at transmitter (CSIT) of MIMO-IDMA [30] and the MUTP [11] [31] has been discussed in literature to support the benefits of MIMO IDMA system.

Thus from the study of the literature, the integration of IDMA with single or dual polarized MIMO system provides greater benefits in terms of the spatial multiplexing and spatial diversity.

The vertical Bell laboratories layered space time (VBLAST) architecture [32] which transmits multiple data streams simultaneously over all antennas, is an example of spatial multiplexing. The VBLAST MIMO can provide higher data rate without increasing the bandwidth and the decoding complexity. However this system is limited by ICI and IAI as discussed earlier. On the other hand, the space time block codes (STBC) [33] [34] are the perfect examples for spatial diversity. The Alamouti orthogonal STBC (OSTBC) for two transmit antennas with full rate is discussed in [33]. However in such a system, the overall performance is affected with increase in the number of antennas due to the correlation properties of the channel due to rich scattering effects and small antenna spacing [35]. Also, for different antenna inclinations, the polarization gain is estimated for both LOS and non-LOS (NLOS) environment [36]. The results showed that, the mobile antenna inclination has minimal effect on the polarization diversity irrespective of the polarization scheme.

The performance of the MIMO using dual polarized antenna has been studied in literature. To further improve the diversity gain without increasing the number of antenna elements, this paper investigates the performance of a triple polarized MIMO-IDMA system over correlated frequency selective channels. The benefits of turbo coding have also been integrated into the proposed system architecture along with Log-maximum a posteriori probability (MAPP) decoding algorithm at the receiver terminal. Thus the proposed architecture considers both extrinsic and intrinsic information to detect the desired user signal. The performance metric such as the bit error rate (BER) for single cell multi-user coded triple polarized MIMO-IDMA system with MMSE receiver is evaluated following the Stanford University Interim (SUI) and Long-term Evolution (LTE) channel specifications.

The rest of the paper is organized as follows: The system configuration is described in Section 2. The detection algorithm is illustrated in Section 3. The performance of the proposed system is discussed in Section 4. Finally the conclusions are drawn in Section 5.

\section{System Configuration}

A single cell multi-user downlink communication that supports $K$ users has been considered in the proposed research work. The transceiver architecture for the proposed single cell multi-user MIMO-IDMA system with triple-polarized antennas is shown in Figure 1.

Each of $K$ user is equipped with $\boldsymbol{N}_{r}$ receive antennas and the base station is equipped with $\boldsymbol{N}_{t}$ transmit antennas. The input bit stream from the proposed configuration be expressed as

$$
\boldsymbol{u}_{k}=\left[\boldsymbol{u}_{k 1}, \boldsymbol{u}_{k 2}, \cdots, \boldsymbol{u}_{k m}\right]^{\mathrm{T}}, k=1,2, \cdots, K .
$$

The bit stream is encoded using the channel encoder. Let $\boldsymbol{b}_{k}$ defines the input bit stream with $\mathrm{m}$ bits after channel encoding and is transmitted to the $k^{\text {th }}$ user through the base station (BS).

The encoded bit stream,

$$
\boldsymbol{b}_{k}=\left[\boldsymbol{b}_{k 1}, \boldsymbol{b}_{k 2}, \cdots, \boldsymbol{b}_{k m}\right]^{\mathrm{T}}, k=1,2, \cdots, K .
$$

To overcome burst errors, interleavers are deployed following the encoder section. Then the bit stream $\boldsymbol{b}_{k j}$, $j=1,2,3, \cdots, m$ is then spreaded using the user-specific orthogonal spreading sequence $\boldsymbol{c}_{k}$ which is given by

$$
\boldsymbol{c}_{k} \equiv\left[c_{k 0}, c_{k 1}, \cdots, c_{k\left(N_{c}-1\right)}\right]^{\mathrm{T}},
$$

where, $N_{c}$ is length of each frames

The element $\boldsymbol{c}_{k}$ is referred as the code bits. $\boldsymbol{c}_{k}$ is permuted by an interleaver $\boldsymbol{\pi}_{k}$,

This user-specific spreading matrix is used to obtain the spreading sequence of the $k^{\text {th }}$ user having the length vector $N_{c} m$ and is expressed as

$$
\boldsymbol{C}_{k} \boldsymbol{b}_{k} \text {, where } k=1,2, \cdots, K \text {. }
$$

All the user's signals are then combined to obtain the final transmission bit stream $\boldsymbol{x}_{k}$ as defined by, 

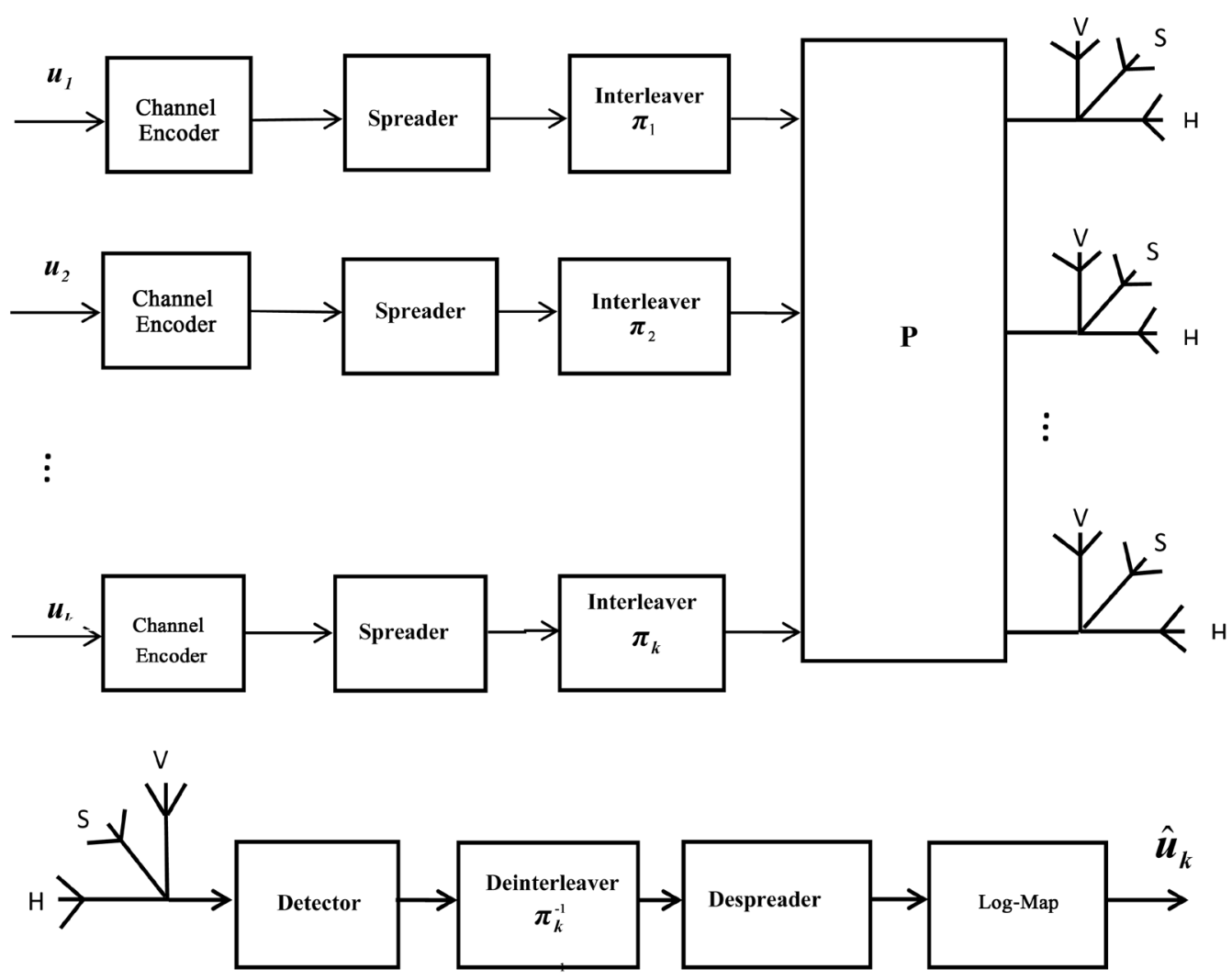

Figure 1. Transceiver structure of single cell multi-user triple-polarized MIMO-IDMA system. V—Vertical; H-Horizontal; S-Slant.

$$
\boldsymbol{x}_{k}=\sum_{k=1}^{K} \boldsymbol{C}_{k} \boldsymbol{b}_{k} .
$$

The bit streams are transmitted using VBLAST architecture using triple-polarized antennas at the transmitter end.

The channel state information matrix of the proposed single triple-polarized antenna at the BS and MS is given by [26] and is represented in Figure 2.

$$
\overline{\overline{\boldsymbol{H}}}_{t p}=\left[\begin{array}{lll}
h_{v v} & h_{v h} & h_{v s} \\
h_{h v} & h_{h h} & h_{h s} \\
h_{s v} & h_{s h} & h_{s s}
\end{array}\right] .
$$

The cross polarization discrimination (XFD) factor estimates the polarization losses incurred in the proposed scheme. The XPD is a random component and it gives the amount of signal loss due to information leakage in adjacent polarization. This leakage is primarily attributed to the losses induced by the channel. Due to rich scattering effects in the mobile environment, the transmitted signal undergoes depolarization. Experiments have proven that antenna orientation that could give higher cross polarization discrimination can be employed to overcome the effects of depolarization. The equation to estimate XPD in a frequency selective channel [14] [26] is given by,

$$
X P D_{v h}=\frac{E\left\{\left|\tilde{h}_{v v}\right|^{2}\right\}}{E\left\{\left|\tilde{h}_{h v}\right|^{2}\right\}},
$$




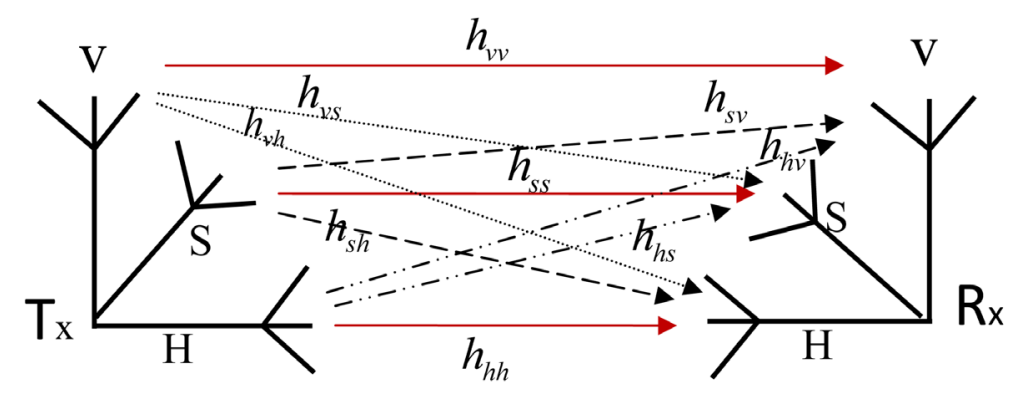

Figure 2. Triple-polarized antenna configuration.

$$
\begin{gathered}
X P D_{h v}=\frac{E\left\{\left|\tilde{h}_{h h}\right|^{2}\right\}}{E\left\{\left|\tilde{h}_{v h}\right|^{2}\right\}}, \\
X P D_{s v}=\frac{E\left\{\left|\tilde{h}_{s s}\right|^{2}\right\}}{E\left\{\left|\tilde{h}_{v s}\right|^{2}\right\}}, \\
X P D_{v s}=\frac{E\left\{\left|\tilde{h}_{v v}\right|^{2}\right\}}{E\left\{\left|\tilde{h}_{s v}\right|^{2}\right\}}, \\
X P D_{h s}=\frac{E\left\{\left|\tilde{h}_{h h}\right|^{2}\right\}}{E\left\{\left|\tilde{h}_{s h}\right|^{2}\right\}}
\end{gathered}
$$

and

$$
X P D_{s h}=\frac{E\left\{\left|\tilde{h}_{s s}\right|^{2}\right\}}{E\left\{\left|\tilde{h}_{h s}\right|^{2}\right\}} .
$$

The $X P D$ is chosen as,

$$
X P D=\frac{1-\left(\beta_{1}+\beta_{2}\right)}{\beta_{1}+\beta_{2}}, 0<\left(\beta_{1}+\beta_{2}\right) \leq 1 .
$$

The $X P D$ for the fixed component matrix of the triply polarized channel [26] is given by

$$
X P D_{f}=\frac{1-\left(\beta_{1 f}+\beta_{2 f}\right)}{\beta_{1 f}+\beta_{2 f}}, 0<\left(\beta_{1 f}+\beta_{2 f}\right) \leq 1
$$

and the normalizations factor is defined by [26] as,

$$
\begin{gathered}
\left|\tilde{h}_{v v}\right|^{2}=\left|\tilde{h}_{h h}\right|^{2}=\left|\tilde{h}_{s s}\right|^{2}=1-\left(\beta_{1 f}+\beta_{2 f}\right) \\
\left|\tilde{h}_{v h}\right|^{2}=\left|\tilde{h}_{s v}\right|^{2}=\left|\tilde{h}_{h s}\right|^{2}=\beta_{1 f} \\
\left|\tilde{h}_{h v}\right|^{2}=\left|\tilde{h}_{v s}\right|^{2}=\left|\tilde{h}_{s h}\right|^{2}=\beta_{2 f} .
\end{gathered}
$$

From the above set of equations, the normalization factor for the triply-polarized antenna [26] can be esti- 
mated as

$$
\begin{gathered}
E\left\{\left|\bar{h}_{v v}\right|^{2}\right\}=E\left\{\left|\bar{h}_{h h}\right|^{2}\right\}=E\left\{\left|\bar{h}_{s s}\right|^{2}\right\}=1-\left(\beta_{1}+\beta_{2}\right) \\
E\left\{\left|\bar{h}_{v h}\right|^{2}\right\}=E\left\{\left|\bar{h}_{s v}\right|^{2}\right\}=E\left\{\left|\bar{h}_{s h}\right|^{2}\right\}=\beta_{1} \\
E\left\{\left|\bar{h}_{h v}\right|^{2}\right\}=E\left\{\left|\bar{h}_{v s}\right|^{2}\right\}=E\left\{\left|\bar{h}_{s h}\right|^{2}\right\}=\beta_{2} .
\end{gathered}
$$

Thus, for the triple-polarized MIMOIDMA system with $N_{r} \times N_{t}$ antennas at the BS and MS, the channel state information matrix is given by

$$
\boldsymbol{H}_{t p}=\rho_{t p} \odot\left(\boldsymbol{R}_{r x}^{1 / 2} \overline{\overline{\boldsymbol{H}}}_{t p} \boldsymbol{R}_{t x}^{1 / 2}\right)
$$

where

$$
\rho_{t p}=\left[\begin{array}{ccc}
\sqrt{1-\mu} & \sqrt{\beta_{1}} & \sqrt{\beta_{2}} \\
\sqrt{\beta_{2}} & \sqrt{1-\mu} & \sqrt{\beta_{1}} \\
\sqrt{\beta_{1}} & \sqrt{\beta_{2}} & \sqrt{1-\mu}
\end{array}\right]
$$

is the polarization leakage matrix with $\mu=\beta_{1}+\beta_{2}$ where $0 \leq \mu \leq 1$ under symmetric conditions. Further based on the investigations through (21), it can be inferred that the correlation matrices at the transmitter and receiver end is defined as $R_{r x}^{1 / 2}$ and $R_{t x}^{1 / 2}$. For an array of spatially distributed triple polarized antennas, from [26] it can be inferred that the channel state information matrix be represented as,

$$
\boldsymbol{H}_{t p}=1_{N_{k} / 3 \times N_{t} / 3} \otimes \rho_{t p} \odot\left(\boldsymbol{R}_{r x}^{1 / 2} \overline{\overline{\boldsymbol{H}}}_{t p N_{k} \times N_{t}} \boldsymbol{R}_{t x}^{1 / 2}\right) .
$$

In the proposed scheme, the channel matrix that connects the $k^{\text {th }}$ MS and BS is assumed to be frequen-

\begin{tabular}{|c|c|c|c|c|c|c|c|c|c|c|}
\hline \multirow{2}{*}{$\begin{array}{l}\text { Path Number } \\
\text { (l) }\end{array}$} & \multicolumn{2}{|c|}{$\begin{array}{l}\text { SUI-1 channel } \\
\text { model }\end{array}$} & \multicolumn{2}{|c|}{$\begin{array}{l}\text { SUI-3 channel } \\
\text { model }\end{array}$} & \multicolumn{2}{|c|}{$\begin{array}{l}\text { SUI-5 channel } \\
\text { model }\end{array}$} & \multicolumn{2}{|c|}{$\begin{array}{l}\text { LTE-extended } \\
\text { vehicular channel } \\
\text { model }\end{array}$} & \multicolumn{2}{|c|}{$\begin{array}{l}\text { LTE-extended } \\
\text { pedestrian } \\
\text { channel model }\end{array}$} \\
\hline & Delay ( $\mu \mathrm{s})$ & $\begin{array}{c}\text { Power (dB) } \\
\quad p\left(\tau_{l}\right)\end{array}$ & $\begin{array}{l}\text { Delay } \\
(\mu \mathrm{s})\end{array}$ & $\begin{array}{c}\text { Power (dB) } \\
\quad p\left(\tau_{l}\right)\end{array}$ & $\begin{array}{c}\text { Delay } \\
(\mu \mathrm{s})\end{array}$ & $\begin{array}{c}\text { Power (dB) } \\
p\left(\tau_{l}\right)\end{array}$ & $\begin{array}{c}\text { Delay } \\
\text { (ns) }\end{array}$ & $\begin{array}{c}\text { Power (dB) } \\
p\left(\tau_{l}\right)\end{array}$ & Delay (ns) & $\begin{array}{l}\text { Power } \\
(\mathrm{dB}) \\
p\left(\tau_{l}\right)\end{array}$ \\
\hline 1 & 0 & 0 & 0 & 0 & 0 & 0 & 0 & 0 & 0 & 0 \\
\hline 2 & 0.4 & -15 & 0.4 & -5 & 4 & -5 & 30 & -1.5 & 30 & -1 \\
\hline 3 & 0.9 & -20 & 0.9 & -10 & 10 & -10 & 150 & 0 & 70 & -2 \\
\hline 4 & & & & & & & 310 & -1.5 & 90 & -3 \\
\hline 5 & & & & & & & 370 & -0.6 & 110 & -8 \\
\hline 6 & & & & & & & 710 & -9.1 & 190 & -8 \\
\hline 7 & & & & & & & 1090 & -7 & 410 & -20.8 \\
\hline 8 & & & & & & & 1730 & -12 & & \\
\hline 9 & & & & & & & 2510 & -16.9 & & \\
\hline
\end{tabular}
cy-selective and the channel model is assumed to satisfy the specifications as stipulated by Standard University Interim (SUI) and Long-Term Evolution (LTE) specifications. Table 1 summarizes the power delay profile of the proposed scheme under SUI and LTE conditions. Equation (24) represents the impulse response connecting $j^{\text {th }}$ receive antenna and $i^{\text {th }}$ transmit antenna is given by

\section{Table 1. Channel specifications for SUI and LTE channel models.}




$$
\boldsymbol{h}_{j i}(t)=\sum_{l=1}^{L} h_{j i}^{l} \partial\left(t-\tau_{l}\right)
$$

where, $L$ represents number of paths between the $j^{\text {th }}$ receive antenna and $i^{\text {th }}$ transmit antenna.

The received signal vector component $y$ at the $k^{\text {th }}$ MS is given by,

$$
\boldsymbol{y}=\boldsymbol{H}_{k t p} \boldsymbol{x}+\boldsymbol{n}_{k}
$$

where $\boldsymbol{H}_{k t p}$ is the triple-polarized $N_{r} \times N_{t}$ channel matrix.

$\boldsymbol{n}_{k}$ is the complex Gaussian noise with $N_{r}$ length vector component and $\boldsymbol{x}$ is the transmitted $N_{r} \times 1$ vector component.

Thus from Equation (25), it can be clearly stated that,

$$
\boldsymbol{y}=\underbrace{\boldsymbol{H}_{k \neq p} \boldsymbol{x}_{k}}_{\substack{\text { Desired } \\ \text { SIgnal }}}+\underbrace{\sum_{i=1, i \neq k}^{K} \boldsymbol{H}_{k p p} \boldsymbol{x}_{i}}_{\text {MAI }}+\boldsymbol{n}_{k}^{\prime}, k=1,2, \cdots, K
$$

where $\boldsymbol{n}_{k}^{\prime}$ is the Gaussian complex vector with zero mean and the covariance matrix is given by

$$
X\left\{\boldsymbol{n}^{\prime}\left(\boldsymbol{n}^{\prime}\right)^{H}\right\}=\sigma^{2} \boldsymbol{I}_{\boldsymbol{N}_{r}}
$$

where $X\{\cdot\}$ and $\sigma$ are the mean and standard deviation.

\section{VBLAST/MIMSE Detection Algorithm}

At the receiver end, minimum mean square estimation (MMSE) detection algorithm using successive interference cancellation technique is considered to estimate the transmitted bit sequence from the triple polarized antennas. The detection algorithm at the receiver is described as follows. The relationship between the transmitted and received bit sequences in a MIMO-IDMA system is given by,

$$
\boldsymbol{y}=\overline{\boldsymbol{H}} \boldsymbol{x}+\boldsymbol{n} .
$$

Initialize

$$
\begin{gathered}
i \leftarrow 1 \\
E_{i}=\left(\boldsymbol{H}^{H} \boldsymbol{H}+\sigma^{2} \boldsymbol{I}_{M}\right)^{-1} \boldsymbol{H}^{H} \\
z_{i}=\arg \min \left\|\left(\mathrm{E}_{i}\right)\right\| .
\end{gathered}
$$

Recursion

$$
\begin{gathered}
\boldsymbol{W}_{z_{i}}=\left(E_{i}\right)_{z_{i}} \\
\boldsymbol{x}_{\bar{z}_{i}}=\left(\boldsymbol{W}_{i}\right)_{z_{i}} \boldsymbol{r}_{i} \\
\boldsymbol{y}_{i+1}=\boldsymbol{y}_{i}-\boldsymbol{x}_{z_{i}}\left(\boldsymbol{H}_{z_{i}}\right) \\
E_{i+1}=\left(\boldsymbol{H}_{\bar{z}_{i}}^{H} \boldsymbol{H}_{\bar{z}_{i}}+\sigma^{2} \boldsymbol{I}_{M}\right)^{-1} \boldsymbol{H}_{\bar{z}_{i}}^{H}, M=4 \\
z_{i+1}=\arg \min \left\|\left(E_{i+1}\right)_{f}\right\| \\
f \notin\left\{z_{1} \cdots z_{i}\right\} \\
i \leftarrow i+1 .
\end{gathered}
$$

Using the algorithm described above, the MMSE detection algorithm estimates the transmitted bit sequence as, $\hat{\boldsymbol{x}}_{k}=\boldsymbol{x}+\boldsymbol{n}$. The estimated bit streams are then deinterleaved using an user specific deinterleaver module to obtain the original bit sequence as estimated by $\tilde{\boldsymbol{b}}_{k}$. $\tilde{\boldsymbol{b}}_{k}$ is then despread following $\hat{\boldsymbol{b}}_{k}=\boldsymbol{S}_{k}^{\mathrm{T}} \cdot \tilde{\boldsymbol{b}}_{k}$. A turbo decoding algorithm based on Log-MAP decoding algorithm is then used at the receiver to obtain $\hat{\boldsymbol{u}}_{k}$. 


\section{Results and Discussion}

This section presents the computer simulation results of the proposed MIMO-IDMA system following the channel standards as specified by the SUI [37] and LTE [38]. The channel model parameters are given in Table 1 and simulation parameters are shown in Table 2.

During simulation, the Doppler shift of 0.5 and $2.5 \mathrm{~Hz}$ are considered with an antenna correlation coefficient of $0.7,0.4$ and 0.3 for SUI-1, SUI-3 and SUI-5 channel models [37] respectively. Under LTE-typical urban channel model and LTE-extended vehicular considerations [38], the Doppler shift is assumed to be $70 \mathrm{~Hz}$ for a mobile moving at a velocity of $40 \mathrm{kmph}$ and $162 \mathrm{kmph}$ respectively. Furthermore, 10,000 channel realizations are made for each SNR. A 1/2 rate parallel concatenated convolution encoder is considered for channel encoding with $\eta=0.4$.

The BER performance of the uncoded and the proposed coded triple-polarized MIMO-IDMA system is shown in Figure 3. in all the cases ten iterations were performed assuming SUI-1 channel model with VBLAST/ MMSE receiver. From the realized results it is evident that, the proposed coded MIMO-IDMA scheme could achieve a $3 \mathrm{~dB}$ better than the uncoded MIMO-IDMA scheme.

Table 2. Simulation parameters.

\begin{tabular}{cc}
\hline Parameters & Attributes \\
\hline Modulation technique & BPSK \\
Channel spacing & $20 \mathrm{MHz}$ \\
Sampling frequency & $22.5 \mathrm{MHz}$ \\
Number of triple-polarized antenna at the transmitter & 1 \\
Number of triple -polarized antenna at the mobile station & 1 \\
Channel model & SUI-1, SUI3, SUI-5 \\
Channel coding & Turbo \\
XPD & -3.98 Db \\
\hline
\end{tabular}

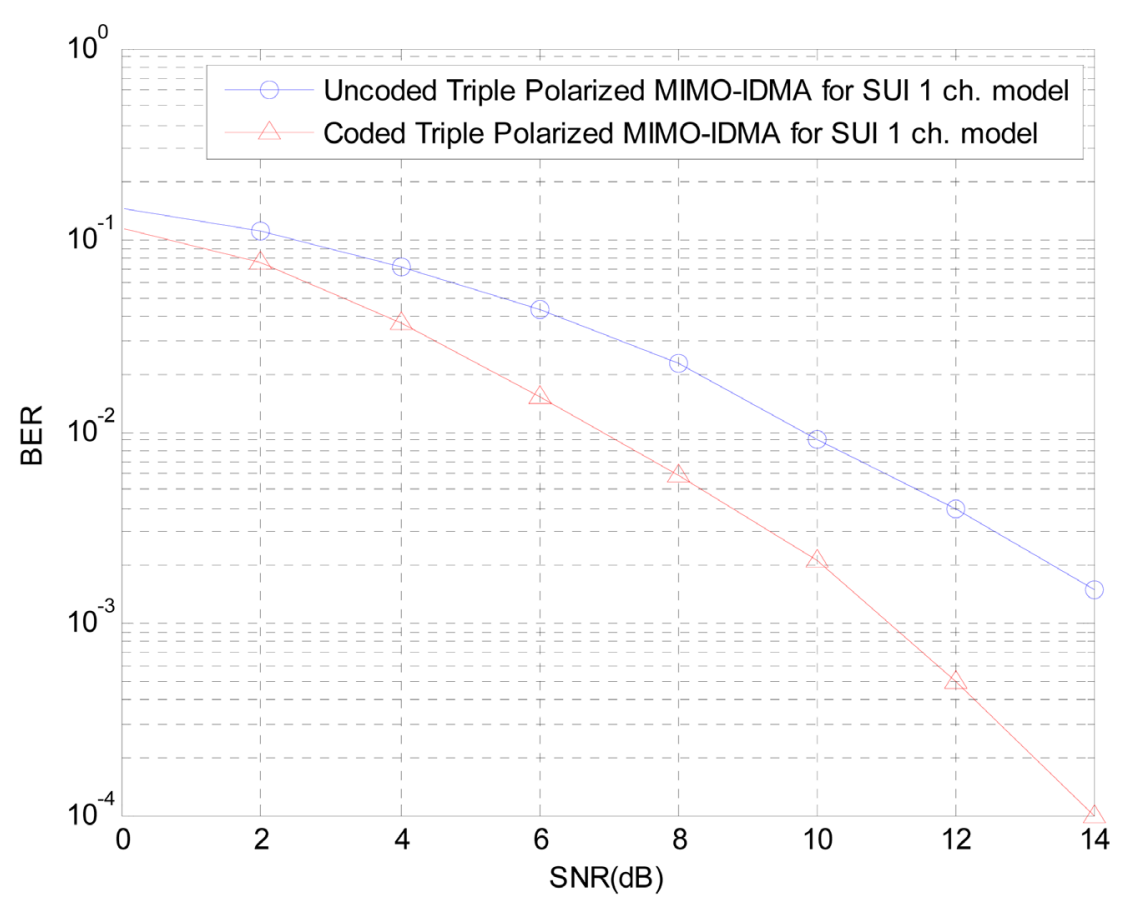

Figure 3. BER versus SNR for coded and uncoded MIMO-IDMA for under SUI 1 channel model. 
The BER performance of uncoded and the proposed triple-polarized MIMO-IDMA system is depicted in Figure 4 and Figure 5 for SUI-3 and SUI-5 channel specifications respectively. From the results it can be inferred that the proposed MIMO-IDMA system could offer better BER at low SNR as compared to the uncoded triple-polarized MIMO-IDMA system.

Figure 6 shows the BER performance of the proposed triple-polarized MIMO-IDMA system under various channel models and the uni-polarized MIMO-IDMA system was compared, the proposed triple polarized MIMO-IDMA system under LTE channel model requires an additional $6 \mathrm{~dB}$ SNR compared to the single polarized antenna case for a fixed BER of $10^{-6}$.

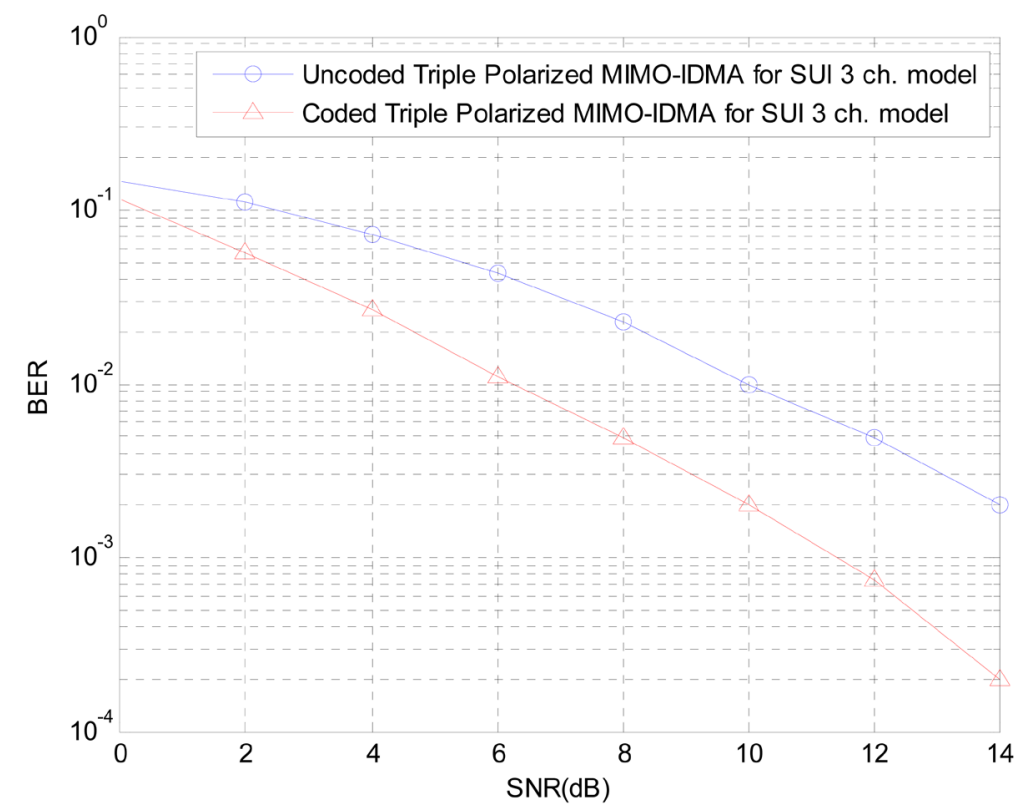

Figure 4. BER versus SNR for coded and uncoded MIMO-IDMA system for SUI 3 channel model.

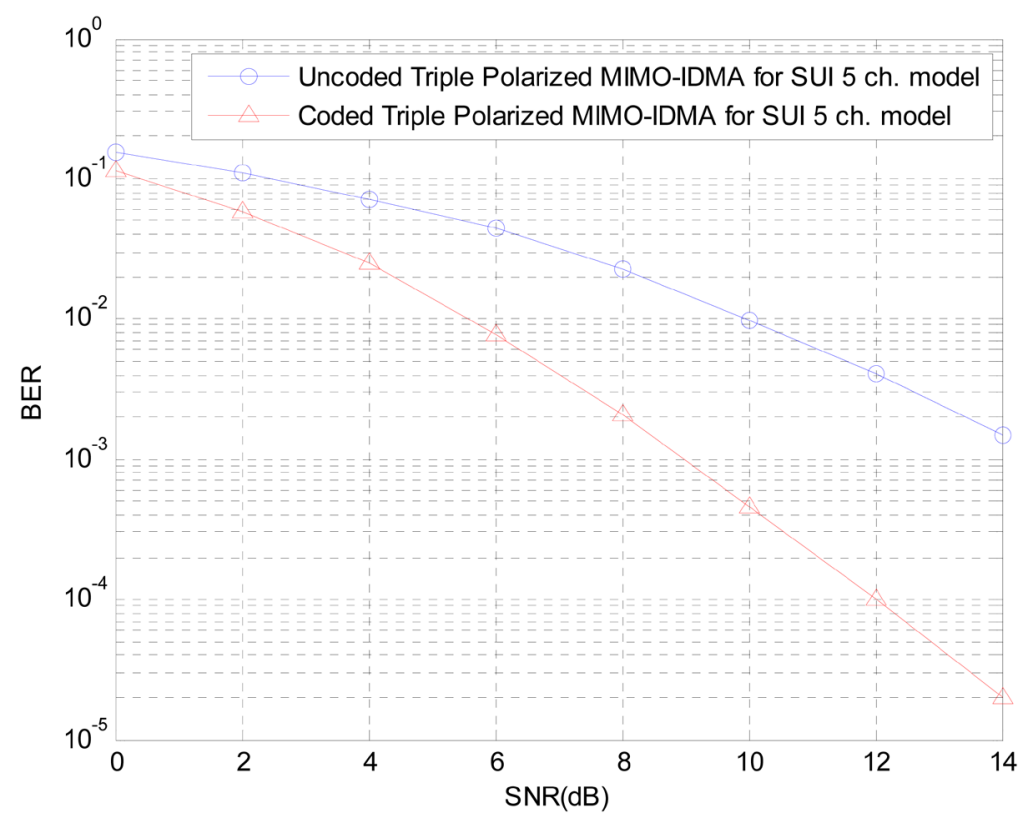

Figure 5. BER versus SNR for coded and uncoded MIMO-IDMA system for SUI 5 channel model. 
The results in Figure 7 indicate that the proposed scheme requires an SNR of $6 \mathrm{~dB}$ to achieve a BER of $10^{-6}$ as compared to uni-polarized MIMO-IDMA system. Thus from the results presented in Figure 6 and Figure 7, it can be concluded that the proposed triple polarized MIMO-IDMA system for LTE channel model equipped with turbo coding can perform better in terms of BER even under rich fading conditions.

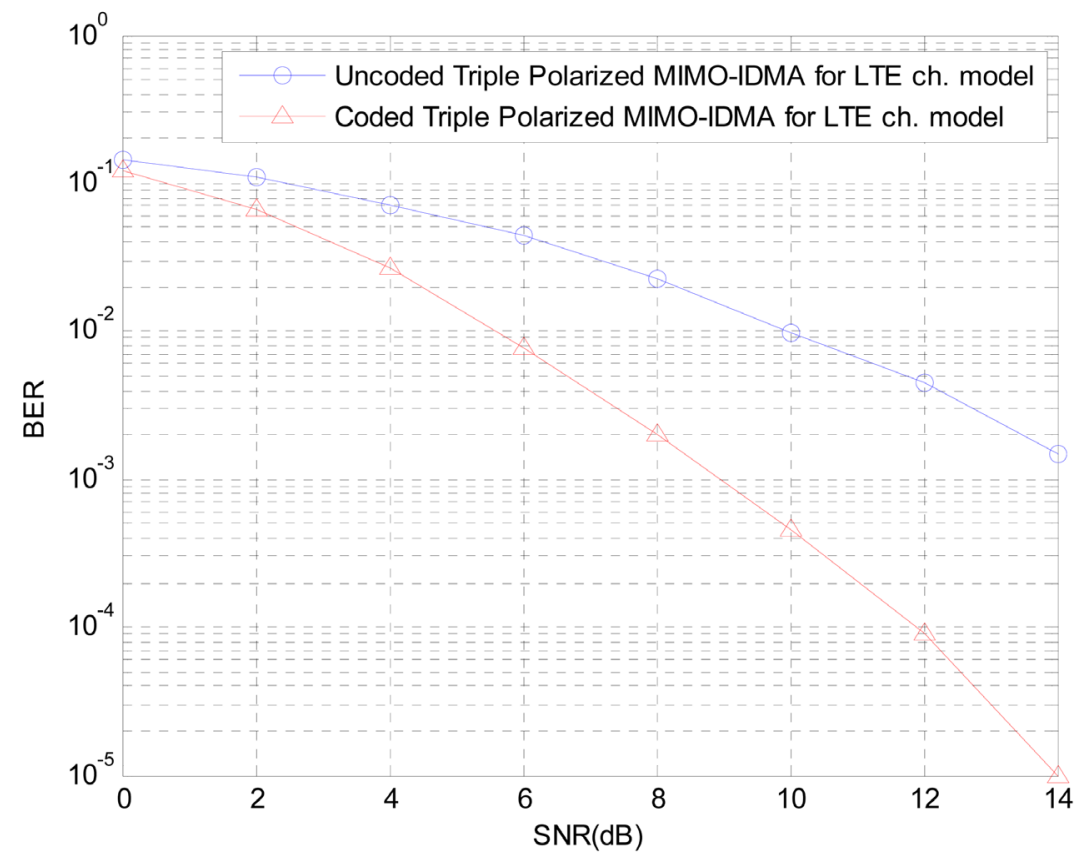

Figure 6. BER versus SNR for coded and uncoded MIMO-IDMA system for LTE channel model.

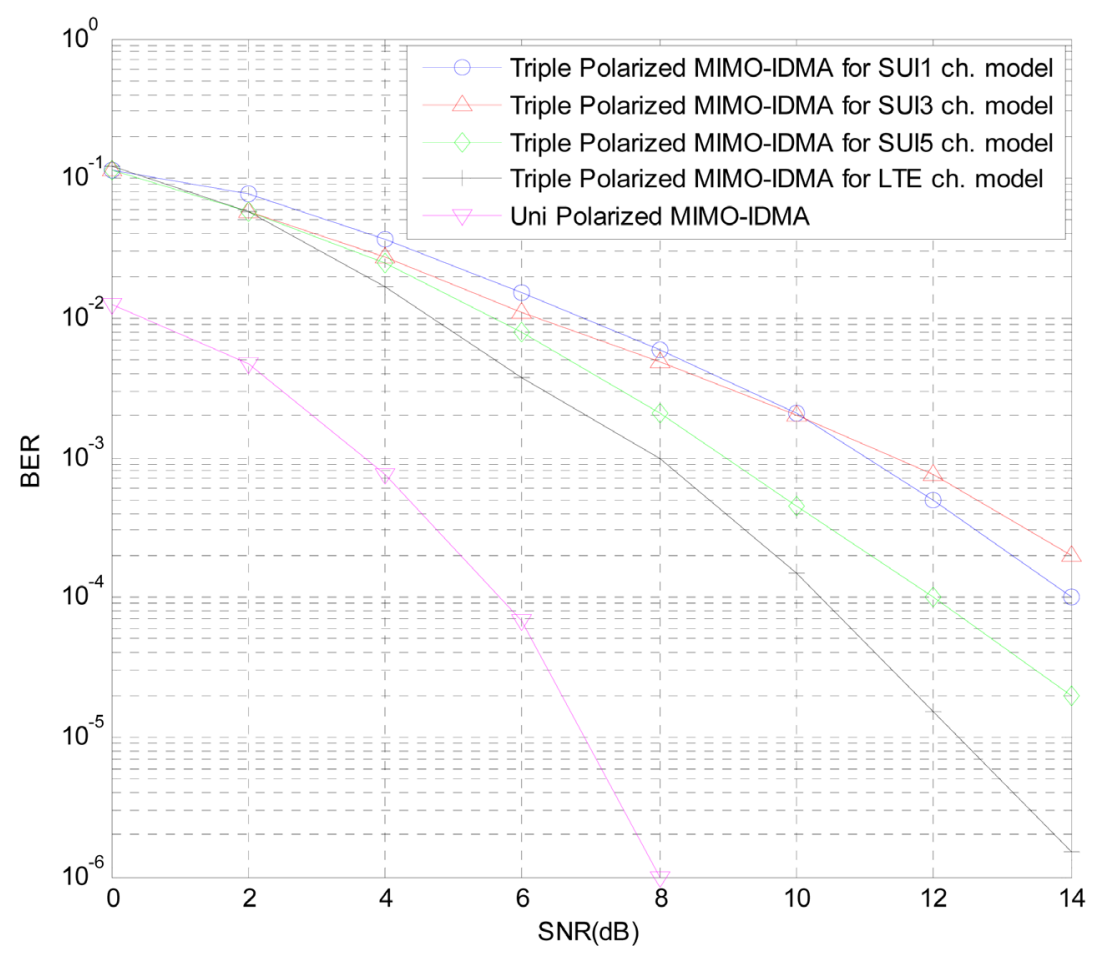

Figure 7. BER versus SNR for MIMO-IDMA system under various channel model. 


\section{Conclusion}

This paper presented a new technique to improve the performance of the existing MIMO-IDMA system. The usage of a triple polarized antenna at the BS and MS has been demonstrated and the performance is analysed using the SUI and LTE channel models. Form the presented results, it can be inferred that the BER performance of the proposed scheme is enhanced in a rich scattering environment characterized by path dependent Doppler and angular spread. The MSI at the transmitter and receiver end is minimized through the usage of the proposed architecture. Thus the proposed triple polarized MIMO-IDMA system with turbo coding performs well at a low SNR offering higher data rate with significant reduction in cost and space requirements for developing the infrastructure.

\section{References}

[1] Oestges, C., Erceg, V. and Paulraj, A.J. (2004) Propagation Modeling of MIMO Multipolarized Fixed Wireless Channels. IEEE Transactions on Vehicular Technology, 53, 644-654. http://dx.doi.org/10.1109/TVT.2004.827149

[2] Foschini, G. and Gans, M. (1998) On Limits of Wireless Communications in a Fading Environment When Using Multiple Antennas. Wireless Personal Communications, 6, 311-335. http://dx.doi.org/10.1023/A:1008889222784

[3] Ping, L., Liu, L., Wu, K. and Leung, W.K. (2006) Interleave-Division Multiple Access. IEEE Transactions on Wireless Communications, 5, 938-947.

[4] Ping, L. (2005) Interleave-Division Multiple Access and Chip-by-Chip Iterative Multi-User Detection. IEEE Communications Magazine, 43, 19-23. http://dx.doi.org/10.1109/MCOM.2005.1452830

[5] Kusume, K. and Bauch, G. (2005) CDMA and IDMA: Iterative Multiuser Detections for Near-Far Asynchronous Communications. 2005 IEEE 16th International Symposium on Personal, Indoor and Mobile Radio Communications, Berlin, Germany, 11-14 September 2005, 426-431. http://dx.doi.org/10.1109/pimrc.2005.1651472

[6] Partibane, B., Nagarajan, V., Vishvaksenan, K.S. and Kalidoss, R. (2015) Performance of Multi-User Transmitter PreProcessing Assisted Multi-Cell IDMA System for Downlink Transmission. Fluctuation and Noise Letters, 14, No. 3. http://dx.doi.org/10.1142/S0219477515500303

[7] Kusume, K., Dietl, G., Utschick, W. and Bauch, G. (2007) Performance of Interleave Division Multiple Access Based on Minimum Mean Square Error Detection. 2007 IEEE International Conference on Communications, Glasgow, U.K., 24-28 June 2007, 2961-2966. http://dx.doi.org/10.1109/icc.2007.492

[8] Vijayarangan, V., Kalidoss, R. and Sukanesh, R. (2006) Low Crest Mapping for PAPR Reduction in OFDM Systems. IET International Conference on Wireless Mobile and Multimedia Networks, Hangzhou, China, 1-4. http://dx.doi.org/10.1049/cp:20061333

[9] Rajakani, K. and Bhagyaveni, M.A. (2012) Adaptive Duplex Technique for Reduction of Turnaround Time in IEEE 802.22. Communications in Computer and Information Science, 269, 503-511. http://dx.doi.org/10.1007/978-3-642-29219-4_57

[10] Kalidoss, R., Bhagyaveni, M.A. and Vishvaksenan, K.S. (2014) A Location Based Duplex Scheme for Cost Effective Rural Broadband Connectivity Using IEEE 802.22 Cognitive Radio Based Wireless Regional Area Networks. Fluctuation and Noise Letters, 13, No. 04. http://dx.doi.org/10.1142/S021947751450028X

[11] Karthipan, R., Vishvaksenan, K.S., Kalidoss, R. and Babu, R.S. (2016) Uplink Capacity Enhancement in IEEE 802.22 Using Modified Duplex Approach. Wireless Personal Communications, 86, 635-656. http://dx.doi.org/10.1007/s11277-015-2949-2

[12] Karthipan, R., Vishvaksenan, K.S., Kalidoss, R. and Krishan, A. (2016) Performance of Turbo Coded Triply-Polarized MIMO-CDMA System for Downlink Communication. Computers and Electrical Engineering. http://dx.doi.org/10.1016/i.compeleceng.2016.01.004

[13] Cristea, B., Roviras, D. and Escrig, B. (2009) Turbo Receivers for Interleave Division Multiple Access Systems. IEEE Transactions on Communications, 57, 2090-2097. http://dx.doi.org/10.1109/TCOMM.2009.07.070525

[14] Nabar, R.U., B’lcskei, H., Erceg, V., et al. (2002) Performance of Multiantenna Signalling Techniques in the Presence of Polarization Diversity. IEEE Transactions on Signal Processing, 50, 2553-2562. http://dx.doi.org/10.1109/TSP.2002.803322

[15] Novak, C., Hlawatsch, F. and Matz, G. (2007) MIMO-IDMA: Uplink Multiuser MIMO Communications Using Interleave-Division Multiple Access and Low-Complexity Iterative Receivers. 2007 IEEE International Conference on Acoustics, Speech and Signal Processing, Honolulu, HI, USA, 15-20 April 2007, 225-228. http://dx.doi.org/10.1109/icassp.2007.366513

[16] Hao, D. and Hoeher, P.A. (2008) Helical Interleaver Set Design for Interleave-Division Multiplexing and Related 
Techniques. IEEE Communications Letters, 12, 843-845. http://dx.doi.org/10.1109/LCOMM.2008.080990

[17] Kusume, K. and Bauch, G. (2008) Simple Construction of Multiple Interleavers: Cyclically Shifting a Single Interleaver. IEEE Transactions on Communications, 56, 1394-1397. http://dx.doi.org/10.1109/TCOMM.2008.060420

[18] Zhou, X., Shi, Z. and Reed, M.C. (2007) Iterative Channel Estimation for IDMA Systems in Time-Varying Channels. IEEE Global Telecommunications Conference, Washington DC, 26-30 November 2007, 4020-4024. http://dx.doi.org/10.1109/glocom.2007.764

[19] Hoeher, P.A., Schoeneich, H. and Fricke, C. (2008) Multi-Layer Interleave-Division Multiple Access: Theory and Practice. European Transactions on Telecommunications, 19, 523-536. http://dx.doi.org/10.1002/ett.1267

[20] Mahafeno, I., Langlais, C. and Jego, C. (2006) OFDM-IDMA versus IDMA with ISI Cancellation for Quasi-Static Rayleigh Fading Multipath Channels. 4th International Symposium on Turbo Codes \& Related Topics, Munich, 3-7 April 2006, 3-7.

[21] Ping, L., Guo, Q. and Tong, J. (2007) The OFDM-IDMA Approach to Wireless Communication Systems. IEEE Wireless Communications, 14, 18-24. http://dx.doi.org/10.1109/MWC.2007.386608

[22] Novak, C., Matz, G. and Hlawatsch, F. (2009) Factor Graph Based Design of an OFDM-IDMA Receiver Performing Joint Data Detection, Channel Estimation, and Channel Length Selection. IEEE International Conference on Acoustics, Speech and Signal Processing, Taipei, 19-24 April 2009, 2561-2564.

[23] Wang, X. and Poor, H.V. (1999) Iterative (Turbo) Soft Interference Cancellation and Decoding for Coded CDMA. IEEE Transactions on Communications, 47, 1046-1061. http://dx.doi.org/10.1109/26.774855

[24] Spencer, Q.H., Swindlehurst, A.L. and Haardt, M. (2004) Zero-Forcing Methods for Downlink Spatial Multiplexing in Multiuser MIMO Channels. IEEE Transactions on Signal Processing, 52, 461-471. http://dx.doi.org/10.1109/TSP.2003.821107

[25] Yang, L.-L. (2008) A Zero-Forcing Multi-User Transmitter Pre-Processing Scheme for Downlink Communications. IEEE Transactions on Communications, 56, 862-865. http://dx.doi.org/10.1109/TCOMM.2008.060354

[26] Habib, A. (2012) Receive Antenna Selection in Diversely Polarized MIMO Transmissions with Convex Optimization. Physical Communication, 5, 328-337. http://dx.doi.org/10.1016/j.phycom.2012.03.003

[27] Liu, W., Yang, L.L. and Hanzo, L. (2008) SVD Assisted Joint Transmitter and Receiver Design for the Downlink of MIMO Systems. IEEE 68th Vehicular Technology Conference (VTC), Calgary, 21-24 September 2008, 1-5. http://dx.doi.org/10.1109/vetecf.2008.284

[28] Boutros, J. and Caire, G. (2002) Iterative Multiuser Joint Decoding: Unified Framework and Asymptotic Analysis. IEEE Transactions on Information Theory, 48, 1772-1793. http://dx.doi.org/10.1109/TIT.2002.1013125

[29] Kschischang, F.R., Frey, B.J. and Loeliger, H.-A. (2001) Factor Graphs and the Sum-Product Algorithm. IEEE Transactions on Information Theory, 47, 498-519. http://dx.doi.org/10.1109/18.910572

[30] Loeliger, H.-A., Dauwels, J., Hu, J., Korl, S., Ping, L. and Kschischang, F.R. (2007) The Factor Graph Approach to Model-Based Signal Processing. Proceedings of the IEEE, 95, 1295-1322. http://dx.doi.org/10.1109/JPROC.2007.896497

[31] Wymeersch, H. (2007) Iterative Receiver Design. Cambridge University Press, New York. http://dx.doi.org/10.1017/CBO9780511619199

[32] Wolniansky, P.W., Foschini, G.J., Golden, G. and Valenzuela, R. (1998) V-Blast: An Architecture for Realizing Very High Data Rates over the Rich-Scattering Wireless Channel. URSI International Symposium on Signals, Systems, and Electronics, Pisa, 29 September-2 October 1998, 295-300.

[33] Alamouti, S. (1998) A Simple Transmit Diversity Technique for Wireless Communications. IEEE Journal on Selected Areas in Communications, 16, 1451-1458. http://dx.doi.org/10.1109/49.730453

[34] Tarokh, V., Jafarkhani, H. and Calderbank, A.R. (1999) Space-Time Block Codes from Orthogonal Designs. IEEE Transactions on Information Theory, 45, 1456-1467. http://dx.doi.org/10.1109/18.771146

[35] Kermoal, J.P., Schumacher, L., Pedersen, K.I., Mogensen, P.E. and Frederiksen, F. (2002) A Stochastic MIMO Radio Channel Model with Experimental Validation. IEEE Journal on Selected Areas in Communications, 20, 1211-1226. http://dx.doi.org/10.1109/JSAC.2002.801223

[36] Lempiainen, J.J. and Laiho-Steffens, J.K. (1998) The Performance of Polarization Diversity Schemes at a Base Station in Small/Micro Cells at 1800 MHz. IEEE Transactions on Vehicular Technology, 47, 1087-1092. http://dx.doi.org/10.1109/25.704863

[37] Maucher, J. and Heise, F.J. (2007) IEEE Std. 2007, IEEE Standard for WIMAX 802.16. Hannover Publisher.

[38] 3GPP (TR 30.803) (2007) Evolved Universal Terrestrial Radio Access (E-UTRA); User Equipment (UE) Radio Transmission and Reception (Release 8). Technical Specification, Sophia Antipolis. 